\title{
A CONTINUATION RESULT FOR DIFFERENTIAL EQUATIONS
}

\author{
T. A. BURTON
}

\begin{abstract}
In this note we show how the Conti-Wintner and Yoshizawa continuation results can be strengthened and combined to produce a flexible continuation theorem. That result is then applied to second and third order equations.
\end{abstract}

1. Introduction. We consider a system of ordinary differential equations

$$
x^{\prime}=f(t, x)
$$

with $f:[0, \infty) \times R^{n} \rightarrow R^{n}$ and continuous. Then for each $\left(t_{0}, x_{0}\right)$ in $[0, \infty) \times$ $R^{n}$ there is at least one solution $x\left(t, t_{0}, x_{0}\right)$ defined on a right-maximal interval $\left[t_{0}, T\right)$, and, if $T<\infty$, then $\lim _{t \rightarrow T^{-}}|x(t)|=+\infty$.

There are two general results yielding $T=\infty$ which have had wide application. The first is known as the Wintner-Conti theorem [1], [4], [5], and the second may be traced back at least to Yoshizawa, but is given in a better form by Lakshmikantham and Leela [3, p. 135].

THEOREM 1. Let $\lambda$ and $\omega$ be continuous functions with $\lambda:[0, \infty) \rightarrow[0, \infty)$, $\omega$ : $[0, \infty) \rightarrow(0, \infty)$, and $\int_{0}^{\infty}[d s / \omega(s)]=\infty$. If $|f(t, x)|<\lambda(t) \omega(|x|)$ on $[0, \infty) \times$ $R^{n}$, then each solution of (1) can be continued for all future time.

The virtue of this result over the next is that the functions $\lambda$ and $\omega$ are often apparent from $f$ itself, while the next result requires construction of a Liapunov function $V$. However, investigators have been very successful in constructing particular Liapunov functions, but these functions frequently fail to fulfill some condition of the result now given.

TheOREM 2. Let $V$ and $\phi$ be continuous functions, $V:[0, \infty) \times R^{n} \rightarrow[0, \infty)$, $V(t, x) \rightarrow \infty$ as $|x| \rightarrow \infty$ uniformly for $t$ in compact sets, $V$ locally Lipschitz in $x, \phi:[0, \infty) \times[0, \infty) \rightarrow(-\infty, \infty)$, and $V_{(1)}^{\prime}(t, x) \leqslant \phi(t, V)$ on $[0, \infty) \times R^{n}$. If for each $u_{0}>0$ the maximal solution of

$$
u^{\prime}=\phi(t, u), \quad u\left(t_{0}\right)=u_{0},
$$

can be continued for all $t \geqslant t_{0}$, then each solution of (1) exists for all future time.

Received by the editors March 17, 1977.

AMS (MOS) subject classifications (1970). Primary 34A15; Secondary 34A40, 34D20.

Key words and phrases. Differential equations, continuation of solutions, differential inequalities, Liapunov functions. 
In examples it frequently happens that $V(t, x) \nrightarrow \infty$ as $|x| \rightarrow \infty$, although $V$ is unbounded along certain paths in $R^{n}$. Also, if $f=\left(f_{1}, \ldots, f_{n}\right)$, then for some $i$ an inequality $\left|f_{i}(t, x)\right|<\lambda(t) \omega(|x|)$ holds for $\lambda$ and $\omega$ satisfying Theorem 1, but for at least one $i$, the inequality fails.

With this in mind we combine the results and obtain a more flexible theorem.

2. An extension. Let $V, g, r, h, \eta$ and $\omega$ be continuous functions with the following properties:

(a) $V:[0, \infty) \times R^{n} \rightarrow[0, \infty), V$ locally Lipschitz in $x$.

(b) $g, h$, and $\eta:[0, \infty) \rightarrow[0, \infty)$.

(c) $r:[0, \infty) \times[0, \infty) \rightarrow(-\infty, \infty)$ and for each $u_{0}>0$ the maximal solution of $\left[u^{\prime}=r(t, u), u\left(t_{0}\right)=u_{0}\right]$ exists for all $t \geqslant t_{0}$.

(d) $V_{(1)}^{\prime}(t, x)<-g(t, x)+r(t, V)$ on $[0, \infty) \times R^{n}$.

(e) $\omega:[0, \infty) \rightarrow(0, \infty), \int_{0}^{\infty}[d s / \omega(s)]=\infty$, and $\omega$ is monotone nondecreasing.

THEOREM 3. Let (a)-(e) hold and suppose that for each $T>0$ and each $i$, if

(A) $\left|f_{i}(t, x)\right| \leqslant \eta(t)[g(t, x)+h(V(t, x))+\omega(|x|)]$ for $0<t<T$ and $x \in$ $R^{n}$ fails, then for each $K>0$ there exists $M>0$ such that either

(B) $V(t, x)<K, 0 \leqslant t \leqslant T$, and $x \in R^{n}$ implies $\left|f_{i}(t, x)\right|<M$, or

(C) $V(t, x)<K$ and $0 \leqslant t \leqslant T$ implies $\left|x_{i}\right| \leqslant M$ holds.

Then each solution of (1) exists for all future time.

Proof. If the theorem is false then there is a solution $x(t)$ of (1) defined on $\left[t_{0}, T\right)$ with $T<\infty$ and $\lim _{t \rightarrow T^{-}}|x(t)|=+\infty$.

Let $u_{0}=V\left(t_{0}, x\left(t_{0}\right)\right)$ in (c) and conclude that $V(t, x(t))<u\left(t, t_{0}, u_{0}\right)$, the maximal solution through $\left(t_{0}, u_{0}\right)$, for $t_{0} \leqslant t<T$. Thus, if $K=$ $\max _{t_{0}<t<T}\left|u\left(t, t_{0}, u_{0}\right)\right|$ then $V(t, x(t)) \leqslant K$ on $\left[t_{0}, T\right)$ and $h(V(t, x(t)))<H$ on $\left[t_{0}, T\right)$ for some $H>0$.

Also, $r$ is continuous and so there exists $Q>0$ with $r(t, V(t, x(t)))<Q$ on $\left[t_{0}, T\right)$. We then have $V^{\prime}(t, x(t)) \leqslant-g(t, x(t))+Q$ so that

$$
\begin{aligned}
0 & \leqslant V(t, x(t)) \\
& \leqslant V\left(t_{0}, x\left(t_{0}\right)\right)+Q\left(T-t_{0}\right)-\int_{t_{0}}^{t} g(s, x(s)) d s,
\end{aligned}
$$

yielding $\int_{t_{0}}^{T^{-}} g(s, x(s)) d s<P$ for some $P>0$.

Next, note that if (C) holds for some $i$, then $\left|x_{i}(t)\right|<M$ on $\left[t_{0}, T\right)$. If (B) holds for some $i$, then

$$
\begin{aligned}
\left|x_{i}(t)\right| & \leqslant\left|x_{i}\left(t_{0}\right)\right|+\int_{t_{0}}^{t}\left|f_{i}(s, x(s))\right| d s \\
& \leqslant\left|x_{i}\left(t_{0}\right)\right|+M\left(T-t_{0}\right) .
\end{aligned}
$$

While if (B) and (C) fail, then (A) yields

$$
\left|x_{i}(t)\right| \leqslant\left|x_{i}\left(t_{0}\right)\right|+N\left[P+H\left(T-t_{0}\right)+\int_{t_{0}}^{t} \omega(|x(s)|) d s\right],
$$


where $N=\max _{t_{0}<t<T} \eta(t)$. Thus, in any of these cases we have

$$
\left|x_{i}(t)\right|<\left|x_{i}\left(t_{0}\right)\right|+S+\int_{t_{0}}^{t} N \omega(|x(s)|) d s
$$

on $\left[t_{0}, T\right)$ for some $S>0$.

We conclude that

$$
|x(t)|<J+L \int_{t_{0}}^{t} \omega(|x(s)|) d s
$$

on $\left[t_{0}, T\right)$ for some positive constants $J$ and $L$. As $\omega$ is monotone nondecreasing, $|x(t)|$ is bounded on $\left[t_{0}, T\right)$ by the maximal solution of

$$
v^{\prime}=L \omega(v), \quad v\left(t_{0}\right)=J
$$

(cf. Hartman [2, p. 29]). However, that solution can be continued for all future time and so $|x(t)|$ is bounded on $\left[t_{0}, T\right)$. This completes the proof.

Remark 1. Efforts to prove Theorem 3 without the monotonicity assumption on $\omega$ have failed, but it seems reasonable that the condition is not needed.

EXAMPLE 1. Consider the system of three equations,

$$
x^{\prime}=y, \quad y^{\prime}=z, \quad z^{\prime}=-\phi(x, y) z-\psi(y)-\gamma(x),
$$

in which we have taken $(x, y, z)=\left(x_{1}, x_{2}, x_{3}\right)$ to avoid subscripts. It is assumed that all functions are continuous, $\phi(x, y)>2 a$ for some $a>0, \gamma^{\prime}(x)$ is bounded, $y \psi(y)>0$ if $y \neq 0, x \gamma(x)>0$ if $x \neq 0$, and $y \partial \phi(x, y) / \partial x<0$.

Let $\Gamma(x)=\int_{0}^{x} \gamma(s) d s, G(y)=\int_{0}^{y} \psi(s) d s$, and assume $a \Gamma(x)+y \gamma(x)+$ $G(y)>-S$ for some $S>0$. Define

and obtain

$$
\begin{aligned}
V(t, x, y, z)= & (z+a y)^{2} / 2+a \Gamma(x)+y \gamma(x) \\
& +G(y)+a \int_{0}^{y}\{\phi(x, s)-a\} s d s+S
\end{aligned}
$$

$$
\begin{aligned}
V^{\prime}= & -\left\{a y \psi(y)-y^{2} \gamma^{\prime}(x)\right\}-\{\phi(x, y)-a\} z^{2} \\
& +a y \int_{0}^{y} s[\partial \phi(x, s) / \partial x] d s \\
< & d y^{2}-a z^{2},
\end{aligned}
$$

as $\gamma^{\prime}(x)$ is bounded and $\phi(x, y)>2 a$. But $\int_{0}^{y}\{\phi(x, s)-a\} s d s>a y^{2} / 2$ and so $V^{\prime}<-a z^{2}+\alpha V$ for some constant $\alpha>0$.

Now $V$ bounded yields $y$ bounded and, hence, $z$ is bounded. Thus, refer to (B) and (C) of Theorem 3 and note that $V(t, x)<K$ yields $f_{1}$ bounded, $f_{2}$ bounded, and $x_{3}$ bounded on an interval $t_{0}<t<T$. Solutions are continuable.

We have taken $g(t, x, y, z)=a z^{2}, h(V(t, x))=0$, and $r(t, s)=\alpha s$. As (A) was not used, it suffices to set $\eta(t)=\omega(s)=1$.

EXAMPLE 2. Again, let $(x, y)=\left(x_{1}, x_{2}\right)$ and consider a system

$$
x^{\prime}=y-\phi(x), \quad y^{\prime}=-\psi(x)+e(t)
$$


with all functions continuous, $\int_{0}^{x} \psi(s) d s>-P$, and $\psi(x) \phi(x)>-Q$ for some positive numbers $P$ and $Q$. We assume that there is a nondecreasing continuous function $\lambda:[0, \infty) \rightarrow(0, \infty)$ with $|\phi(x)|<\psi(x) \phi(x)+P+\lambda(|x|)$, where $\int_{0}^{\infty}[d s /\{s+\lambda(s)\}]=\infty$.

Take $V=\left[y^{2} / 2\right]+\int_{0}^{x} \psi(s) d s+P$ and obtain

$$
\begin{aligned}
V^{\prime} & =-\psi(x) \phi(x)+y e(t) \\
& \leqslant-[\psi(x) \phi(x)+Q]+2|e(t)| V+Q+|e(t)| .
\end{aligned}
$$

Then $r(t, s)=2|e(t)| s+|e(t)|+Q, \quad g(t, x)=\psi(x) \phi(x)+Q, \quad \eta(t)=1$, and $h(s)=0$.

When $V<K$, then $y=x_{2}$ is bounded. We have

$$
\begin{aligned}
\left|f_{1}(t, x)\right| & =|y-\phi(x)| \leqslant|y|+|\phi(x)| \\
& \leqslant|y|+\psi(x) \phi(x)+P+\lambda(|x|) \\
& \leqslant \psi(x) \cdot \phi(x)+P+\omega(|x|+|y|),
\end{aligned}
$$

where $\omega(s)=s+\lambda(s)$.

As an instance of Example 2, consider the forced van der Pol equation

$$
\begin{aligned}
& x^{\prime}=y-\varepsilon\left(\left[x^{3} / 3\right]-x\right) \quad(\varepsilon>0), \\
& y^{\prime}=-x+e(t) .
\end{aligned}
$$

We have $\phi(x) \psi(x)=\varepsilon x^{2}\left(\left[x^{2} / 3\right]-1\right)$ so that $\lambda(|x|)=\varepsilon$ will suffice.

REMARK 2. The reader may note that Theorem 1 is often stated with $|f(t, x)|<\omega(t,|x|)$, where solutions of $r^{\prime}=\omega(t, r)$ are continuable. This change can also be made in Theorem 3. Also, in applications it is sometimes conceptually easier to require Condition (A) of Theorem 3 only for those $x$ for which $V(t, x)<K$; that change is also valid.

RemarK 3 . Theorem 3 is fully applicable to delay differential equations

$$
x^{\prime}=f(t, x)+g(t, x(t-\tau(t)))
$$

with $\tau(t)>0$. For if one supposes a solution is defined on an interval $\left[t_{0}, T\right)$ with $\varlimsup_{t \rightarrow T^{-}}|x(t)|=+\infty$, then $x(t-\tau(t))$ is a bounded function on $\left[t_{0}, T\right]$ so that one is considering the continuation problem for the ordinary differential equation

$$
x^{\prime}=f(t, x)+e(t)
$$

for $t_{0}<t<T$ with $e(t)=g(t, x(t-\tau(t)))$. Our Example 2 is an instance of this.

\section{REFERENCES}

1. R. Conti, Limitazioni "in ampiezza" della soluzioni di un sistema di equazioni differenziali e applicazioni, Boll. Un. Mat. Ital. (3) 11 (1956), 344-349. MR 18, 309.

2. P. Hartman, Ordinary differential equations, Wiley, New York, 1964. 
3. V. Lakohmikantham and S. Leela, Differential and integral inequalities, Vol. 1, Academic Preas, New York, 1969.

4. A. Wintmer, The non-local existence problem of ordinany differential equations, Amer. J. Math. 07 (1945), 277-284.

5. $\longrightarrow$, The infinities in the non-local existence problem of ordinany differential equations, Amer. J. Math. 9 (1940), 173-178.

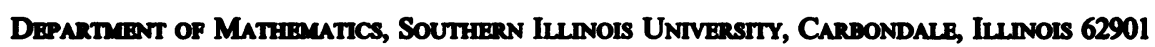

